\title{
INTERVAL CRITERIA FOR FORCED OSCILLATION OF DIFFERENTIAL EQUATIONS WITH $p$-LAPLACIAN AND NONLINEARITIES GIVEN BY RIEMANN-STIELTJES INTEGRALS
}

\author{
Taher S. Hassan And Qingkai Kong
}

\begin{abstract}
We consider forced second order differential equation with $p$-Laplacian and nonlinearities given by a Riemann-Stieltjes integrals in the form of

$$
\left(p(t) \phi_{\gamma}\left(x^{\prime}(t)\right)\right)^{\prime}+q_{0}(t) \phi_{\gamma}(x(t))+\int_{0}^{b} q(t, s) \phi_{\alpha(s)}(x(t)) d \zeta(s)=e(t),
$$

where $\phi_{\alpha}(u):=|u|^{\alpha} \operatorname{sgn} u, \gamma, b \in(0, \infty), \alpha \in C[0, b)$ is strictly increasing such that $0 \leq \alpha(0)<\gamma<\alpha(b-), p, q_{0}, e \in C\left(\left[t_{0}, \infty\right), \mathbb{R}\right)$ with $p(t)>0$ on $\left[t_{0}, \infty\right), q \in C([0, \infty) \times[0, b))$, and $\zeta:[0, b) \rightarrow \mathbb{R}$ is nondecreasing. Interval oscillation criteria of the El-Sayed type and the Kong type are obtained. These criteria are further extended to equations with deviating arguments. As special cases, our work generalizes, unifies, and improves many existing results in the literature.
\end{abstract}

\section{Introduction}

We are concerned with the oscillatory behavior of forced second order differential equations with $p$-Laplacian and nonlinearities given by a RiemannStieltjes integrals in the form of

$$
\left(p(t) \phi_{\gamma}\left(x^{\prime}(t)\right)\right)^{\prime}+q_{0}(t) \phi_{\gamma}(x(t))+\int_{0}^{b} q(t, s) \phi_{\alpha(s)}(x(t)) d \zeta(s)=e(t)
$$

where $\phi_{\alpha}(u):=|u|^{\alpha} \operatorname{sgn} u, \gamma, b \in(0, \infty), \alpha \in C[0, b)$ is strictly increasing such that $0 \leq \alpha(0)<\gamma<\alpha(b-), p, q_{0}, e \in C\left(\left[t_{0}, \infty\right), \mathbb{R}\right)$ with $p(t)>0$ on $\left[t_{0}, \infty\right)$, $q \in C([0, \infty) \times[0, b))$, and $\zeta:[0, b) \rightarrow \mathbb{R}$ is nondecreasing. Our interest is to establish oscillation criteria for Eq.(1.1) without assuming that $q_{0}(t), q(t, s)$, and $e(t)$ are of definite sign. Here $\int_{0}^{b} f(s) d \zeta(s)$ denotes the Riemann-Stieltjes integral of the function $f$ on $[0, b)$ with respect to $\zeta$.

Received March 30, 2011; Revised December 20, 2011.

2010 Mathematics Subject Classification. 34C10, 34C15.

Key words and phrases. interval criteria, forced oscillation, $p$-Laplacian, nonlinear differential equations.

The second author is supported by the NNSF of China (No. 10971231). 
We note that as special cases, the integral term in the equation becomes a finite sum when $\zeta(s)$ is a step function and a Riemann integral when $\zeta(s)=s$.

As usual, a solution $x(t)$ of Eq.(1.1) is said to be oscillatory if it is defined on some ray $[T, \infty)$ with $T \geq 0$, and has unbounded set of zeros. Eq.(1.1) is said to be oscillatory if every solution extendible throughout $\left[t_{x}, \infty\right)$ for some $t_{x} \geq 0$ is oscillatory.

In the last 50 years, there has been extensive work on oscillation and nonoscillation of various differential equations, see [1,3-11, 13, 16-30,32-39] and the references cited therein.

Sun and Wong [35] investigated the following forced equation with mixed nonlinearities

$$
\left(p(t) x^{\prime}(t)\right)^{\prime}+q_{0}(t) x(t)+\sum_{j=0}^{N} q_{j}(t) \phi_{\alpha j}(x(t))=e(t),
$$

where $p, q_{0}$ and $e$ satisfy the same assumptions as for Eq.(1.1), $q_{j} \in C[0, \infty)$ and

$$
\alpha_{1}>\alpha_{2}>\cdots>\alpha_{m}>1>\alpha_{m+1}>\cdots>\alpha_{N}>0 .
$$

Without imposing a restriction on the forcing term $e(t)$ given by Kartsatos and others, see [16,17], that $e(t)$ is the second derivative of an oscillatory function. Hassan, Erbe and Peterson [14] discussed the oscillation of an equation with $p$-Laplacian, more specifically, they established oscillation criteria of El-Sayedtype for the equation

$$
\left(p(t)\left(x^{\prime}(t)\right)^{\gamma}\right)^{\prime}+q_{0}(t) x^{\gamma}(t)+\sum_{j=1}^{N} q_{j}(t) \phi_{\alpha j}(x(t))=e(t)
$$

where $\phi_{\alpha}(u):=|u|^{\alpha} \operatorname{sgn} u, \gamma$ is a quotient of odd positive integers and $\alpha_{j}>0$, $j=1,2, \ldots, N$, such that

$$
\alpha_{1}>\alpha_{2}>\cdots>\alpha_{m}>\gamma>\alpha_{m+1}>\cdots>\alpha_{N}>0 .
$$

Hassan and Kong [15] considered the forced second order differential equations with $p$-Laplacian in the form of

$$
\left(p(t) \phi_{\gamma}\left(x^{\prime}(t)\right)\right)^{\prime}+\sum_{j=0}^{N} q_{j}(t) \phi_{\alpha j}(x(t))=e(t),
$$

where $\gamma, \alpha_{j}>0, j=0,1,2, \ldots, N$, such that

$$
\alpha_{j}>\gamma, j=1,2, \ldots, l ; \text { and } \alpha_{j}<\gamma, j=l+1, l+2, \ldots, N .
$$

They established oscillation criteria of El-Sayed-type and Kong-type for Eq. (1.2). Recently, Sun and Kong [31] considered the equation (1.1) when $\gamma=1$. Motivated by the above, in this paper, we will establish interval oscillation criteria of both the El-Sayed-type and the Kong-type for the more general equation (1.1). Clearly, our work is of significance because Eq.(1.1) not only 
contains a $p$-Laplacian term but also allows an infinite number of nonlinear terms and even a continuum of nonlinearities determined by the function $\zeta$.

Finally, we will extend the results for Eq.(1.1) to forced nonlinear differential equations with deviating arguments in the form

$$
\left(p(t) \phi_{\gamma}\left(x^{\prime}(t)\right)\right)^{\prime}+q_{0}(t) \phi_{\gamma}(x(t))+\int_{0}^{b} q(t, s) \phi_{\alpha(s)}(x(g(t, s))) d \zeta(s)=e(t),
$$

where, in addition to the assumptions for Eq. (1.1), $g \in C([0, \infty) \times[0, b),[0, \infty))$ such that $\lim _{t \rightarrow \infty} g(t, s)=\infty$ for $s \in[0, b)$.

This paper is organized as follows: after this introduction, we state our main results for Eqs.(1.1) and (1.4) in Section 2. All proofs are given in Section 3.

\section{Main results}

We denote by $L_{\zeta}(0, b)$ the set of Riemann-Stieltjes integrable functions on $[0, b)$ with respect to $\zeta$. Let $a \in(0, b)$ such that $\alpha(a)=\gamma$. We further assume that

$$
\alpha^{-1} \in L_{\zeta}(0, b) \quad \text { such that } \int_{0}^{a} d \zeta(s)>0 \text { and } \int_{a}^{b} d \zeta(s)>0 .
$$

We see that the condition $\alpha^{-1} \in L_{\zeta}(0, b)$ is satisfied if either $\alpha(0)>0$ or $\alpha(s) \rightarrow 0$ "slowly" as $s \rightarrow 0^{+}$, or $\zeta(s)$ is constant in a right neighborhood of 0 . To state our main results, we begin with the following lemma which generalizes [31, Lemma 1].

Lemma 2.1. Let

$$
m:=\gamma\left(\int_{a}^{b} d \zeta(s)\right)^{-1} \int_{a}^{b} \alpha^{-1}(s) d \zeta(s)
$$

and

$$
n:=\gamma\left(\int_{0}^{a} d \zeta(s)\right)^{-1} \int_{0}^{a} \alpha^{-1}(s) d \zeta(s) .
$$

Then for any $\delta \in(m, n)$, there exists $\eta \in L_{\zeta}(0, b)$ such that $\eta(s)>0$ on $[0, b)$,

$$
\int_{0}^{b} \alpha(s) \eta(s) d \zeta(s)=\gamma \quad \text { and } \quad \int_{0}^{b} \eta(s) d \zeta(s)=\delta .
$$

We note from the definition of $m$ and $n$ that $0<m<1<n$. In the following, we will use the values of $\delta$ in the interval $(m, 1]$ to establish interval criteria for oscillation of Eq.(1.1). Our first result provides an oscillation criterion of the El-Sayed-type.

Theorem 2.1. Suppose that for any $T \geq 0$ and for $i=1,2$, there exist constants $a_{i}$ and $b_{i}$ with $T \leq a_{i}<b_{i}$ and $\left(a_{1}, b_{1}\right) \cap\left(a_{2}, b_{2}\right)=\emptyset$ such that, for $i=1,2$

$$
q(t, s) \geq 0 \text { for }(t, s) \in\left[a_{i}, b_{i}\right] \times[0, b)
$$


and

$$
(-1)^{i} e(t) \geq 0 \text { for } t \in\left[a_{i}, b_{i}\right] .
$$

Assume further that for $i=1,2$, there exists $u_{i} \in C^{1}\left[a_{i}, b_{i}\right]$ satisfying $u_{i}\left(a_{i}\right)=$ $u_{i}\left(b_{i}\right)=0$ and $u_{i}(t) \not \equiv 0$ on $\left[a_{i}, b_{i}\right]$ such that

$$
\sup _{\delta \in(m, 1]} \int_{a_{i}}^{b_{i}}\left[Q(t)\left|u_{i}(t)\right|^{\gamma+1}-p(t)\left|u_{i}^{\prime}(t)\right|^{\gamma+1}\right] d t>0,
$$

where

$$
Q(t):=q_{0}(t)+\left[\frac{|e(t)|}{1-\delta}\right]^{1-\delta} \exp \left(\int_{0}^{b} \eta(s) \ln \frac{q(t, s)}{\eta(s)} d \zeta(s)\right)
$$

with $\eta(s)$ implicitly defined as in Lemma 2.1 based on $\delta$. Here we use the convention that $\ln 0=-\infty, e^{-\infty}=0$, and $0^{1-\delta}=1$ and $(1-\delta)^{1-\delta}=1$ for $\delta=1$. Then Eq.(1.1) is oscillatory.

Remark 2.1. (i) We observe that in Theorem 2.1, if the supremum in (2.4) is assumed at $\delta=1$, the effect of $e(t)$ is neglected in some extent. This implies that the magnitude of $e(t)$ in $\left[a_{i}, b_{i}\right]$ cannot be large. For otherwise, the supremum would have been taken at some $\delta \in(m, 1)$.

(ii) Contrast to the results in the literature, by choosing different values of $\gamma$, Eq.(1.1) allows the nonlinearities of the unknown function in the integral term to be all sublinear, all superlinear, or mixed.

Following Philos [24], Kong [19], and Kong [20], we say that for any $a, b \in \mathbb{R}$ such that $a<b$, a function $H(t, s)$ belongs to a function class $\mathcal{H}(a, b)$, denoted by $H \in \mathcal{H}(a, b)$, if $H \in C(\mathbb{D}, \mathbb{R})$, where $\mathbb{D}:=\{(t, s): b \geq t \geq s \geq a\}$, which satisfies

$$
H(t, t)=0, \quad H(b, s)>0 \text { and } H(s, a)>0 \text { for } b>s>a,
$$

and $H(t, s)$ has continuous partial derivatives $\partial H(t, s) / \partial t$ and $\partial H(t, s) / \partial s$ on $[a, b] \times[a, b]$ such that

$$
\frac{\partial H(t, s)}{\partial t}=(\gamma+1) h_{1}(t, s) H^{\frac{\gamma}{\gamma+1}}(t, s)
$$

and

$$
\frac{\partial H(t, s)}{\partial s}=(\gamma+1) h_{2}(t, s) H^{\frac{\gamma}{\gamma+1}}(t, s),
$$

where $h_{1}, h_{2} \in L_{l o c}(\mathbb{D}, \mathbb{R})$. Next, we use the function class $\mathcal{H}(a, b)$ to establish an oscillation criterion for Eq.(1.1) of the Kong-type.

Theorem 2.2. Suppose that for any $T \geq 0$ and for $i=1,2$, there exist constants $a_{i}$ and $b_{i}$ with $T \leq a_{i}<b_{i}$ such that (2.2) and (2.3) hold. Assume further that for $i=1,2$, there exists $c_{i} \in\left(a_{i}, b_{i}\right)$ and $H_{i} \in \mathcal{H}\left(a_{i}, b_{i}\right)$ such that

$$
\sup _{\delta \in(m, 1]}\left\{\frac{1}{H_{i}\left(c_{i}, a_{i}\right)} \int_{a_{i}}^{c_{i}}\left[Q(s) H_{i}\left(s, a_{i}\right)-p(s)\left|h_{i 1}\left(s, a_{i}\right)\right|^{\gamma+1}\right] d s\right.
$$




$$
\left.+\frac{1}{H_{i}\left(b_{i}, c_{i}\right)} \int_{c_{i}}^{b_{i}}\left[Q(s) H_{i}\left(b_{i}, s\right)-p(s)\left|h_{i 2}\left(b_{i}, s\right)\right|^{\gamma+1}\right] d s\right\}>0,
$$

where $Q(t)$ is defined by (2.5). Then Eq.(1.1) is oscillatory.

Remark 2.2. (i) When $\gamma=1$, Theorems 2.1 and 2.2 unify and improve Theorems 2.1 and 2.2 in [31].

(ii) For $N \in \mathbb{N}$ and $s \in[0, N+1)$ we let

$$
\zeta(s)=\sum_{j=1}^{N} \chi(s-j) \quad \text { with } \quad \chi(s)= \begin{cases}1, & s \geq 0 \\ 0, & s<0\end{cases}
$$

$\alpha \in C[0, N+1)$ such that $\alpha(j)=\alpha_{j}, j=0,1, \ldots, N$, satisfying (1.3); and $q(t, j)=q_{j}(t) \in C[0, \infty)$ for $j=0,1, \ldots, N$. Then by a simple computation, we see that Lemma 2.1 and Theorems 2.1 and 2.2 reduce to Lemma 2.1, and Theorems 2.1 and 2.2 in [15], respectively.

In the following, we state the interval oscillation criteria for Eq.(1.4).

Theorem 2.3. Assume

$$
g_{*}(t):=\inf _{s \in[0, b)}\{t, g(t, s)\} \text { and } g^{*}(t):=\sup _{s \in[0, b)}\{t, g(t, s)\}
$$

exist. Suppose that for any $T \geq 0$ and for $i=1,2$, there exist constants $a_{i}, b_{i} \in[T, \infty)$ with $T \leq a_{i}<b_{i}$ such that

$$
\begin{gathered}
q_{0}(t) \geq 0 \quad \text { for } t \in\left[g_{*}\left(a_{i}\right), g^{*}\left(b_{i}\right)\right], \\
q(t, s) \geq 0 \quad \text { for }(t, s) \in\left[g_{*}\left(a_{i}\right), g^{*}\left(b_{i}\right)\right] \times[0, b),
\end{gathered}
$$

and

$$
(-1)^{i} e(t) \geq 0, \text { for } t \in\left[g_{*}\left(a_{i}\right), g^{*}\left(b_{i}\right)\right] .
$$

Assume further that there exists $u \in C^{1}\left[a_{i}, b_{i}\right]$ satisfying $u\left(a_{i}\right)=u\left(b_{i}\right)=0$, $i=1,2, u(t) \not \equiv 0$ on $\left[a_{i}, b_{i}\right]$ such that for $i=1,2$,

$$
\sup _{\delta \in(m, 1]} \int_{a_{i}}^{b_{i}}\left[Q_{i}(t)\left|u_{i}(t)\right|^{\gamma+1}-p(t)\left|u_{i}^{\prime}(t)\right|^{\gamma+1}\right] d t>0
$$

where

$$
Q_{i}(t):=q_{0}(t)+\left[\frac{|e(t)|}{1-\delta}\right]^{1-\delta} \exp \left(\int_{0}^{b} \eta(s) \ln \frac{q(t, s) \psi_{i}^{\alpha(s)}(t, s)}{\eta(s)} d \zeta(s)\right)
$$

and

$$
\psi_{i}(t, s):= \begin{cases}\delta_{i}(t, s), & g(t, s)<t \\ 1, & g(t, s)=t \\ \zeta_{i}(t, s), & g(t, s)>t\end{cases}
$$


with

and

$$
\delta_{i}(t, s):=\int_{g\left(a_{i}, s\right)}^{g(t, s)} \frac{d s}{p^{\frac{1}{\gamma}}(s)}\left(\int_{g\left(a_{i}, s\right)}^{t} \frac{d s}{p^{\frac{1}{\gamma}}(s)}\right)^{-1}
$$

$$
\zeta_{i}(t, s):=\int_{g(t, s)}^{g\left(b_{i}, s\right)} \frac{d s}{p^{\frac{1}{\gamma}}(s)}\left(\int_{t}^{g\left(b_{i}, s\right)} \frac{d s}{p^{\frac{1}{\gamma}}(s)}\right)^{-1}
$$

and with $\eta_{j}$ as in Lemma 2.1 based on $\delta$. Here we use the convention that $0^{1-\delta}=1$ and $(1-\delta)^{1-\delta}=1$ when $\delta=1$. Then Eq.(1.4) is oscillatory.

Theorem 2.4. Suppose that for any $T \geq 0$ and for $i=1,2$, there exist constants $a_{i}$ and $b_{i}$ with $T \leq a_{i}<b_{i}$ such that (2.10)-(2.12) hold. Assume further that there exist $c_{i} \in\left(a_{i}, b_{i}\right)$ and $H_{i} \in \mathcal{H}\left(a_{i}, b_{i}\right)$ such that

$$
\begin{aligned}
\sup _{\delta \in(m, 1]}\{ & \frac{1}{H_{i}\left(c_{i}, a_{i}\right)} \int_{a_{i}}^{c_{i}}\left[Q_{i}(s) H_{i}\left(s, a_{i}\right)-p(s)\left|h_{i 1}\left(s, a_{i}\right)\right|^{\gamma+1}\right] d s \\
& \left.\quad+\frac{1}{H_{i}\left(b_{i}, c_{i}\right)} \int_{c_{i}}^{b_{i}}\left[Q_{i}(s) H_{i}\left(b_{i}, s\right)-p(s)\left|h_{i 2}\left(b_{i}, s\right)\right|^{\gamma+1}\right] d s\right\}>0,
\end{aligned}
$$

where $Q_{i}(t)$ is defined by (2.13). Then Eq.(1.4) is oscillatory.

\section{Proofs}

Proof of Lemma 2.1. Let

$$
\eta_{1}(s):= \begin{cases}0, & s \in(0, a) \\ \gamma \alpha^{-1}(s)\left(\int_{a}^{b} d \zeta(s)\right)^{-1}, & s \in[a, b)\end{cases}
$$

and

$$
\eta_{2}(s):= \begin{cases}\gamma \alpha^{-1}(s)\left(\int_{0}^{a} d \zeta(s)\right)^{-1}, & s \in(0, a) \\ 0, & s \in[a, b) .\end{cases}
$$

Clearly for $i=1,2, \eta_{i} \in L_{\zeta}(0, b)$ and

$$
\int_{0}^{b} \alpha(s) \eta_{i}(s) d \zeta(s)=\gamma
$$

Moreover,

For $k \in[0,1]$ let

$$
\int_{0}^{b} \eta_{1}(s) d \zeta(s)=m \text { and } \int_{0}^{b} \eta_{2}(s) d \zeta(s)=n .
$$

$$
\eta(s, k):=(1-k) \eta_{1}(s)+k \eta_{2}(s), \quad s \in[0, b) .
$$

Then it is easy to see that

$$
\int_{0}^{b} \alpha(s) \eta(s, k) d \zeta(s)=\gamma
$$


Furthermore, since $\eta(s, 0)=\eta_{1}(s)$ and $\eta(s, 1)=\eta_{2}(s)$, we have

$$
\int_{0}^{b} \eta(s, 0) d \zeta(s)=m \text { and } \int_{0}^{b} \eta(s, 1) d \zeta(s)=n .
$$

By the continuous dependence of $\eta(s, k)$ on $k$ there exists $k^{*} \in(0,1)$ such that $\eta(s):=\eta\left(s, k^{*}\right)$ satisfies that

$$
\int_{0}^{b} \eta(s) d \zeta(s)=\delta
$$

Note that $\eta(s)>0$ for $s \in[0, b)$ and $\int_{0}^{b} \alpha(s) \eta(s) d \zeta(s)=\gamma$.

The next lemma is a generalized Arithmetic-Geometric mean inequality established in [31].

Lemma 3.1. Let $u \in C[0, b)$ and $\eta \in L_{\zeta}(0, b)$ satisfying $u \geq 0, \eta>0$ on $[0, b)$ and $\int_{0}^{b} \eta(s) d \zeta(s)=1$. Then

$$
\int_{0}^{b} \eta(s) u(s) d \zeta(s) \geq \exp \left(\int_{0}^{b} \eta(s) \ln [u(s)] d \zeta(s)\right)
$$

where we use the convention that $\ln 0=-\infty$ and $e^{-\infty}=0$.

Proof of Theorem 2.1. Assume Eq.(1.1) has an extendible solution $x(t)$ which is eventually positive or negative. Then, without loss of generality, assume $x(t)>0$ for all $t \geq T \geq 0$, where $T$ depends on the solution $x(t)$. When $x(t)$ is an eventually negative, the proof follows the same way except that the interval $\left[a_{2}, b_{2}\right]$, instead of $\left[a_{1}, b_{1}\right]$, is used. Define

$$
z(t):=\frac{p(t) \phi_{\gamma}\left(x^{\prime}(t)\right)}{\phi_{\gamma}(x(t))}, t \geq T \text {. }
$$

It follows from (1.1) that for $t \geq T, z(t)$ satisfies the first order nonlinear Riccati equation

$(3.2) z^{\prime}(t)=-q_{0}(t)-\int_{0}^{b} q(t, s)[x(t)]^{\alpha(s)-\gamma} d \zeta(s)+e(t) x^{-\gamma}(t)-\frac{\gamma|z(t)|^{\frac{\gamma+1}{\gamma}}}{p^{\frac{1}{\gamma}}(t)}$.

From the assumption, there exists a nontrivial interval $\left[a_{1}, b_{1}\right] \subset[T, \infty)$ such that (2.2) and (2.3) hold with $i=1$.

(I) We first consider the case where the supremum in (2.4) is assumed at $\delta=1$. From (2.3) and (3.2), we have that for $t \in\left[a_{1}, b_{1}\right]$

$$
z^{\prime}(t) \leq-q_{0}(t)-\int_{0}^{b} q(t, s)[x(t)]^{\alpha(s)-\gamma} d \zeta(s)-\frac{\gamma|z(t)|^{\frac{\gamma+1}{\gamma}}}{p^{\frac{1}{\gamma}}(t)}
$$


Let $\eta \in L_{\zeta}(0, b)$ be defined as in Lemma 2.1 with $\delta=1$. Then $\eta$ satisfies $(2.1)$ with $\delta=1$. This follows that

$$
\int_{0}^{b} \eta(s)[\alpha(s)-\gamma] d \zeta=0
$$

Then, from Lemma 3.1, we get, for $t \in\left[a_{1}, b_{1}\right]$

$$
\begin{aligned}
& \int_{0}^{b} q(t, s)[x(t)]^{\alpha(s)-\gamma} d \zeta(s) \\
= & \int_{0}^{b} \eta(s) \frac{q(t, s)}{\eta(s)}[x(t)]^{\alpha(s)-\gamma} d \zeta(s) \\
\geq & \exp \left(\int_{0}^{b} \eta(s) \ln \left(\frac{q(t, s)}{\eta(s)}[x(t)]^{\alpha(s)-\gamma}\right) d \zeta(s)\right) \\
= & \exp \left(\int_{0}^{b} \eta(s) \ln \left[\frac{q(t, s)}{\eta(s)}\right] d \zeta(s)+\ln (x(t)) \int_{0}^{b} \eta(s)[\alpha(s)-\gamma] d \zeta(s)\right) \\
= & \exp \left(\int_{0}^{b} \eta(s) \ln \left[\frac{q(t, s)}{\eta(s)}\right] d \zeta(s)\right) .
\end{aligned}
$$

This together with (3.3) shows that

$$
z^{\prime}(t) \leq-Q(t)-\frac{\gamma|z(t)|^{\frac{\gamma+1}{\gamma}}}{p^{\frac{1}{\gamma}}(t)} \quad \text { for } t \in\left[a_{1}, b_{1}\right],
$$

where $Q(t)$ is defined by (2.5) with $\delta=1$. Multiplying both sides of (3.4) by $\left|u_{1}(t)\right|^{\gamma+1}$, integrating from $a_{1}$ to $b_{1}$, and using integration by parts, we find that

$$
\begin{aligned}
& \int_{a_{1}}^{b_{1}} Q(t)\left|u_{1}(t)\right|^{\gamma+1} d t \\
\leq & \int_{a_{1}}^{b_{1}}\left[(\gamma+1) \phi_{\gamma}\left(u_{1}(t)\right) u_{1}^{\prime}(t) z(t)-\frac{\gamma\left|u_{1}(t)\right|^{\gamma+1}}{p^{\frac{1}{\gamma}}(t)}|z(t)|^{\frac{\gamma+1}{\gamma}}\right] d t \\
\leq & \int_{a_{1}}^{b_{1}}\left[(\gamma+1)\left|u_{1}(t)\right|^{\gamma}\left|u_{1}^{\prime}(t)\right||z(t)|-\frac{\gamma\left|u_{1}(t)\right|^{\gamma+1}}{p^{\frac{1}{\gamma}}(t)}|z(t)|^{\frac{\gamma+1}{\gamma}}\right] d t .
\end{aligned}
$$

Let $\lambda:=\frac{\gamma+1}{\gamma}$. Define $A$ and $B$ by

$$
A^{\alpha}:=\frac{\gamma\left|u_{1}(t)\right|^{\gamma+1}}{p^{\frac{1}{\gamma}}(t)}|z(t)|^{\lambda} \text { and } B^{\alpha-1}:=\left|u_{1}^{\prime}(t)\right|(\gamma p(t))^{\frac{1}{\gamma+1}}
$$

Using the inequality in [12] we have

$$
\alpha A B^{\alpha-1}-A^{\alpha} \leq(\alpha-1) B^{\alpha},
$$


i.e.,

$$
(\gamma+1)\left|u_{1}^{\prime}(t)\right|\left|u_{1}(t)\right|^{\gamma}|z(t)|-\frac{\gamma\left|u_{1}(t)\right|^{\gamma+1}}{p^{\frac{1}{\gamma}}(t)}|z(t)|^{\lambda} \leq p(t)\left|u_{1}^{\prime}(t)\right|^{\gamma+1},
$$

which together with (3.5) implies that

$$
\int_{a_{1}}^{b_{1}} Q(t)\left|u_{1}(t)\right|^{\gamma+1} d t \leq \int_{a_{1}}^{b_{1}} p(t)\left|u_{1}^{\prime}(t)\right|^{\gamma+1} d t
$$

This leads to a contradiction to (2.4).

(II) Now, we consider the case where the supremum in (2.4) is assumed at $\delta \in(m, 1)$. Then from $(2.3)$ we see that for $t \in\left[a_{1}, b_{1}\right]$

$$
z^{\prime}(t)=-q_{0}(t)-\int_{0}^{b} q(t, s)[x(t)]^{\alpha(s)-\gamma} d \zeta(s)-|e(t)| x^{-\gamma}(t)-\frac{\gamma|z(t)|^{\frac{\gamma+1}{\gamma}}}{p^{\frac{1}{\gamma}}(t)} .
$$

Let $\widetilde{\eta}(s)=\delta^{-1} \eta(s)$. Then from (2.1) we have

$$
\int_{0}^{b} \widetilde{\eta}(s) d \zeta(s)=1 \quad \text { and } \quad \int_{0}^{b} \widetilde{\eta}(s)[\delta \alpha(s)-\gamma] d \zeta=0 .
$$

Hence for $t \in\left[a_{1}, b_{1}\right]$

$$
\begin{aligned}
& \int_{0}^{b} q(t, s)[x(t)]^{\alpha(s)-\gamma} d \zeta(s)+|e(t)| x^{-\gamma}(t) \\
= & \int_{0}^{b} \widetilde{\eta}(s)\left(\delta \eta^{-1}(s) q(t, s)[x(t)]^{\alpha(s)-\gamma}+|e(t)| x^{-\gamma}(t)\right) d \zeta(s) .
\end{aligned}
$$

Using the Arithmetic-Geometric mean inequality, see [2, Page 17],

$$
c h+d k \geq c^{h} d^{k}, \quad \text { where } c, d \geq 0, h, k>0 \text { and } h+k=1,
$$

with

$$
c=\eta^{-1}(s) q(t, s)[x(t)]^{\alpha(s)-\gamma}, d=\frac{1}{1-\delta}|e(t)| x^{-\gamma}(t), h=\delta \text { and } k=1-\delta,
$$

we have that for $t \in\left[a_{1}, b_{1}\right]$ and $s \in[0, b)$

$$
\begin{aligned}
& \delta \eta^{-1}(s) q(t, s)[x(t)]^{\alpha(s)-\gamma}+(1-\delta) \frac{|e(t)|}{1-\delta} x^{-\gamma}(t) \\
\geq & {\left[\frac{q(t, s)}{\eta(s)}\right]^{\delta}\left[\frac{|e(t)|}{1-\delta}\right]^{1-\delta}[x(t)]^{\delta \alpha(s)-\gamma} . }
\end{aligned}
$$

Substituting this into (3.9) and using Lemma 3.1 and (3.8), we see that for $t \in\left[a_{1}, b_{1}\right]$

$$
\int_{0}^{b} q(t, s)[x(t)]^{\alpha(s)-\gamma} d \zeta(s)+|e(t)| x^{-\gamma}(t)
$$




$$
\begin{aligned}
& \geq \exp \left(\int_{0}^{b} \tilde{\eta}(s) \ln \left(\left[\frac{q(t, s)}{\eta(s)}\right]^{\delta}\left[\frac{|e(t)|}{1-\delta}\right]^{1-\delta}[x(t)]^{\delta \alpha(s)-\gamma}\right) d \zeta(s)\right) \\
& =\exp \left(\int_{0}^{b} \widetilde{\eta}(s)\left(\ln \left[\frac{q(t, s)}{\eta(s)}\right]^{\delta}+\ln \left[\frac{|e(t)|}{1-\delta}\right]^{1-\delta}+[\delta \alpha(s)-\gamma] \ln x(t)\right) d \zeta(s)\right) \\
& =\left[\frac{|e(t)|}{1-\delta}\right]^{1-\delta} \exp \left(\int_{0}^{b} \eta(s) \ln \frac{q(t, s)}{\eta(s)} d \zeta(s)\right)
\end{aligned}
$$

It follows from (3.7) and (3.10) that for $t \in\left[a_{1}, b_{1}\right]$

$$
\begin{aligned}
z^{\prime}(t) & \leq-q_{0}(t)-\left[\frac{|e(t)|}{1-\delta}\right]^{1-\delta} \exp \left(\int_{0}^{b} \eta(s) \ln \frac{q(t, s)}{\eta(s)} d \zeta(s)\right)-\frac{\gamma|z(t)|^{\frac{\gamma+1}{\gamma}}}{p^{\frac{1}{\gamma}}(t)} \\
(3.11) & =-Q(t)-\frac{\gamma|z(t)|^{\frac{\gamma+1}{\gamma}}}{p^{\frac{1}{\gamma}}(t)},
\end{aligned}
$$

where $Q(t)$ is defined by $(2.5)$ with $\delta \in(m, 1)$. The rest of the proof is similar to Part (I) and hence is omitted.

Proof of Theorem 2.2. Assume Eq.(1.1) has an extendible solution $x(t)$ which is eventually positive or negative. Then, without loss of generality, assume $x(t)>0$ for all $t \geq T \geq 0$, where $T$ depends on the solution $x(t)$. Define $z(t)$ by (3.1). From (3.4) and (3.11), we get that

$$
z^{\prime}(t) \leq-Q(t)-\frac{\gamma|z(t)|^{\frac{\gamma+1}{\gamma}}}{p^{\frac{1}{\gamma}}(t)} .
$$

Multiplying both sides of (3.12), with $t$ replaced by $s$, by $H_{1}\left(b_{1}, s\right)$ and integrating with respect to $s$ from $c_{1}$ to $b_{1}$, we find that

$$
\begin{aligned}
& \int_{c_{1}}^{b_{1}} Q(s) H_{1}\left(b_{1}, s\right) d s \\
\leq & -\int_{c_{1}}^{b_{1}} z^{\prime}(s) H_{1}\left(b_{1}, s\right) d s-\int_{c_{1}}^{b_{1}} \frac{\gamma|z(t)|^{\frac{\gamma+1}{\gamma}}}{p^{\frac{1}{\gamma}}(t)} H_{1}\left(b_{1}, s\right) d s .
\end{aligned}
$$

Using integration by parts and from (2.6) and (2.8), we obtain that

$$
\begin{aligned}
& \int_{c_{1}}^{b_{1}} Q(s) H_{1}\left(b_{1}, s\right) d s \\
\leq & z\left(c_{1}\right) H_{1}\left(b_{1}, c_{1}\right)+\int_{c_{1}}^{b_{1}}\left[(\gamma+1) h_{12}\left(b_{1}, s\right) H_{1}^{\frac{\gamma}{\gamma+1}}\left(b_{1}, s\right) z(s)\right. \\
& \left.-\frac{\gamma|z(s)|^{\frac{\gamma+1}{\gamma}} H_{1}\left(b_{1}, s\right)}{p^{\frac{1}{\gamma}}(t)}\right] d s
\end{aligned}
$$




$$
\begin{aligned}
\leq & z\left(c_{1}\right) H_{1}\left(b_{1}, c_{1}\right)+\int_{c_{1}}^{b_{1}}\left[(\gamma+1)\left|h_{12}\left(b_{1}, s\right)\right| H_{1}^{\frac{\gamma}{\gamma+1}}\left(b_{1}, s\right)|z(s)|\right. \\
& \left.-\frac{\gamma|z(s)|^{\frac{\gamma+1}{\gamma}} H_{1}\left(b_{1}, s\right)}{p^{\frac{1}{\gamma}}(t)}\right] d s .
\end{aligned}
$$

Let $\lambda=\frac{\gamma+1}{\gamma}$. Define $A$ and $B$ by

$$
A^{\alpha}:=\frac{\gamma|z(s)|^{\lambda} H_{1}\left(b_{1}, s\right)}{p^{\frac{1}{\gamma}}(t)} \text { and } B^{\alpha-1}:=(\gamma p(s))^{\frac{1}{\gamma+1}}\left|h_{12}\left(b_{1}, s\right)\right| .
$$

Then, using the inequality (3.6), we get that

$$
\begin{aligned}
& (\gamma+1)\left|h_{12}\left(b_{1}, s\right)\right| H_{1}^{\frac{\gamma}{\gamma+1}}\left(b_{1}, s\right)|z(s)|-\frac{\gamma|z(s)|^{\frac{\gamma+1}{\gamma}} H_{1}\left(b_{1}, s\right)}{p^{\frac{1}{\gamma}}(s)} \\
\leq & p(s)\left|h_{12}\left(b_{1}, s\right)\right|^{\gamma+1} .
\end{aligned}
$$

This together with (3.13) shows that

$$
\frac{1}{H_{1}\left(b_{1}, c_{1}\right)} \int_{c_{1}}^{b_{1}}\left[Q(s) H_{1}\left(b_{1}, s\right)-p(s)\left|h_{12}\left(b_{1}, s\right)\right|^{\gamma+1}\right] d s \leq z\left(c_{1}\right) .
$$

Similarly, multiplying both sides of (3.12), with $t$ replaced by $s$, by $H_{1}\left(s, a_{1}\right)$ and integrating by parts from $a_{1}$ to $c_{1}$, we see that

$$
\frac{1}{H_{1}\left(c_{1}, a_{1}\right)} \int_{a_{1}}^{c_{1}}\left[Q(s) H_{1}\left(s, a_{1}\right)-p(s)\left|h_{11}\left(s, a_{1}\right)\right|^{\gamma+1}\right] d s \leq-z\left(c_{1}\right) .
$$

Combining (3.14) and (3.15) we get that

$$
\begin{gathered}
\frac{1}{H_{1}\left(c_{1}, a_{1}\right)} \int_{a_{1}}^{c_{1}}\left[Q(s) H_{1}\left(s, a_{1}\right)-p(s) h_{11}^{\gamma+1}\left(s, a_{1}\right)\right] d s \\
+\frac{1}{H_{1}\left(b_{1}, c_{1}\right)} \int_{c_{1}}^{b_{1}}\left[Q(s) H_{1}\left(b_{1}, s\right)-p(s) h_{12}^{\gamma+1}\left(b_{1}, s\right)\right] d s \leq 0 .
\end{gathered}
$$

This contradicts (2.9) with $i=1$.

The following lemma, which was established in [15], plays a key role in the proof of the oscillation criteria for Eq.(1.4).

Lemma 3.2. Suppose that for any $T \geq 0$ and for $i=1,2$, there exist constants $a_{i}$ and $b_{i}$ with $T \leq a_{i}<b_{i}$ such that (2.10)-(2.12) hold. Assume Eq.(1.4) has a nonoscillatory solution $x(t)$ on $[0, \infty)$. Then for $t \in\left[a_{i}, b_{i}\right]$ with $i=1,2$,

$$
\frac{x(g(t, s))}{x(t)} \geq \psi_{i}(t, s) \quad \text { for } i=1,2,
$$

where $\psi_{i}(t, s)$ is defined by $(2.14)$. 
Proof of Theorems 2.3 and 2.4. Without loss of generality, we may assume $x(t), x(g(t, s))>0$ for all $s \in[0, b)$ and $t \geq T \geq 0$. Define $z(t)$ by (3.1). Then

$$
z^{\prime}(t)=-q_{0}(t)-\int_{0}^{b} q(t, s) \frac{x^{\alpha(s)}(g(t, s))}{x^{\gamma}(t)} d \zeta(s)+e(t) x^{-\gamma}(t)-\frac{\gamma|z(t)|^{\frac{\gamma+1}{\gamma}}}{p^{\frac{1}{\gamma}}(t)} .
$$

From the assumption, there exist constants $a_{1}$ and $b_{1}$ with $a_{1}<b_{1}$ and $\left[g_{*}\left(a_{1}\right), g^{*}\left(b_{1}\right)\right] \subset\left[t_{0}, \infty\right)$ such that (2.10)-(2.12) hold with $i=1$. Then from Lemma 3.2 we have that for $t \in\left[a_{1}, b_{1}\right]$ and $j=0,1,2, \ldots, N$

$$
\frac{x^{\alpha(s)}(g(t, s))}{x^{\gamma}(t)} \geq \psi_{1}^{\alpha(s)}(t, s)[x(t)]^{\alpha(s)-\gamma} .
$$

The rest of the proof is similar to those of Theorem 2.1 and 2.2, and is hence omitted.

\section{References}

[1] R. P. Agarwal, S. R. Grace, and D. O'Regan, Oscillation Theory for Second Order Linear, Half-Linear, Superlinear and Sublinear Dynamic Equations, Kluwer Academic, Dordrecht, 2002.

[2] E. F. Beckenbach and R. Bellman, Inequalities, Springer, Berlin, 1961.

[3] G. J. Butler, Oscillation theorems for a nonlinear analogue of Hill's equation, Quart. J. Math. Oxford Ser. (2) 27 (1976), no. 106, 159-171.

[4] _ Integral averages and the oscillation of second order ordinary differential equations, SIAM J. Math. Anal. 11 (1980), no. 1, 190-200.

[5] D. Çakmak and A. Tiryaki, Oscillation criteria for certain forced second order nonlinear differential equations with delayed argument, Comput. Math. Appl. 49 (2005), no. 11-12, 1647-1653.

[6] C. V. Coffman and J. S. W. Wong, Oscillation and nonoscillation of solutions of generalized Emden-Fowler equations, Trans. Amer. Math. Soc. 167 (1972), 399-434.

[7] E. M. Elabbasy and T. S. Hassan, Interval oscillation for second order sublinear differential equations with a damping term, Int. J. Dyn. Syst. Differ. Equ. 1 (2008), no. 4, 291-299.

[8] E. M. Elabbasy, T. S. Hassan, and S. H. Saker, Oscillation of second-order nonlinear differential equations with a damping term, Electron. J. Differential Equations 2005 (2005), No. 76, 13 pp.

[9] M. A. El-Sayed, An oscillation criterion for a forced second order linear differential equation, Proc. Amer. Math. Soc. 118 (1993), no. 3, 813-817.

[10] L. Erbe, T. S. Hassan, and A. Peterson, Oscillation of second order neutral delay differential equations, Adv. Dyn. Syst. Appl. 3 (2008), no. 1, 53-71.

[11] A. F. Güvenilir and A. Zafer, Second order oscillation of forced functional differential equations with oscillatory potentials, Comput. Math. Appl. 51 (2006), no. 9-10, 13951404.

[12] G. H. Hardy, J. E. Littlewood, and G. Polya, Inequalities, Second ed., Cambridge University Press, Cambridge, 1988.

[13] T. S. Hassan, Interval oscillation for second order nonlinear differential equations with a damping term, Serdica Math. J. 34 (2008), no. 4, 715-732.

[14] T. S. Hassan, L. Erbe, and A. Peterson, Forced oscillation of second order functional differential equations with mixed nonlinearities, Acta Mathematica Scientia 31B (2011), no. $2,613-626$. 
[15] T. S. Hassan and Q. Kong, Interval criteria for forced oscillation of differential equations with p-Laplacian, damping, and mixed nonlinearities, Dynamic Systems \& Applications 20 (2011), 279-294.

[16] A. G. Kartsatos, On the maintenance of oscillations of $n$th order equations under the effect of a small forcing term, J. Differential Equations 10 (1971), 355-363.

[17] Maintenance of oscillations under the effect of a periodic forcing term, Proc. Amer. Math. Soc. 33 (1972), 377-383.

[18] M. S. Keener, On the solutions of certain linear nonhomogeneous second-order differential equations, Applicable Anal. 1 (1971), no. 1, 57-63.

[19] Q. Kong, Interval criteria for oscillation of second-order linear ordinary differential equations, J. Math. Anal. Appl. 229 (1999), no. 1, 258-270.

[20] - Oscillation criteria for second order half-linear differential equations, Differential equations with applications to biology (Halifax, NS, 1997), 317-323, Fields Inst. Commun., 21, Amer. Math. Soc., Providence, RI, 1999.

[21] Q. Kong and J. S. W. Wong, Oscillation of a forced second order differential equations with a deviating argument, Funct. Differ. Equ. 17 (2010), no. 1-2, 141-155.

[22] Q. Kong and B. G. Zhang, Oscillation of a forced second order nonlinear equation, Chinese Ann. Math. Ser. B 15 (1994), no. 1, 59-68.

[23] M. K. Kwong and J. S. W. Wong, Linearization of second order nonlinear oscillation theorems, Trans. Amer. Math. Soc. 279 (1983), no. 2, 705-722.

[24] A. H. Nasr, Sufficient conditions for the oscillation of forced super-linear second order differential equations with oscillatory potential, Proc. Amer. Math. Soc. 126 (1998), no. $1,123-125$.

[25] C. H. Ou and J. S. W. Wong, Forced oscillation of nth-order functional differential equations, J. Math. Anal. Appl. 262 (2001), no. 2, 722-731.

[26] Ch. G. Philos, Oscillation theorems for linear differential equations of second order, Arch. Math. (Basel) 53 (1989), no. 5, 482-492.

[27] S. M. Rankin, Oscillation theorems for second order nonhomogeneous linear differential equations, J. Math. Anal. Appl. 53 (1976), no. 3, 550-553.

[28] A. Skidmore and J. J. Bowers, Oscillatory behavior of solutions of $y^{\prime \prime}+p(x) y=f(x)$, J. Math. Anal. Appl. 49 (1975), 317-323.

[29] A. Skidmore and W. Leighton, On the differential equation $y^{\prime \prime}+p(x) y=f(x)$, J. Math. Anal. Appl. 43 (1973), 46-55.

[30] Y. G. Sun, A note on Nasr's and Wong's papers, J. Math. Anal. Appl. 286 (2003), no. $1,363-367$.

[31] Y. G. Sun and Q. Kong, Interval criteria for forced oscillation with nonlinearities given by Riemann-Stieltjes integrals, Comput. Math. Appl. 62 (2011), no. 1, 243-252.

[32] Y. G. Sun and F. W. Meng, Interval criteria for oscillation of second order differential equations with mixed nonlinearities, Appl. Math. Comp. 198 (2008), no. 1, 375-381.

[33] Y. G. Sun, C. H. Ou, and J. S. W. Wong, Interval oscillation theorems for a linear second-order differential equation, Comput. Math. Appl. 48 (2004), no. 10-11, 16931699.

[34] Y. G. Sun and J. S. W. Wong, Note on forced oscillation of nth-order sublinear differential equations, J. Math. Anal. Appl. 298 (2004), no. 1, 114-119.

[35] _ Oscillation criteria for second order forced ordinary differential equations with mixed nonlinearities, J. Math. Anal. Appl. 334 (2007), no. 1, 549-560.

[36] H. Teufel, Forced second order nonlinear oscillations, J. Math. Anal. Appl. 40 (1972), $148-152$.

[37] J. S. W. Wong, Second order nonlinear forced oscillations, SIAM J. Math. Anal. 19 (1988), no. 3, 667-675.

[38] - Oscillation criteria for a forced second-order linear differential equation, J. Math. Anal. Appl. 231 (1999), no. 1, 235-240. 
[39] Q. Yang, Interval oscillation criteria for a forced second order nonlinear ordinary differential equations with oscillatory potential, Appl. Math. Comput. 136 (2003), no. 1, 49-64.

TAHer S. Hassan

Department of Mathematics

FACULTY OF SCIENCE

Mansoura University

Mansoura, 35516, Egypt

E-mail address: tshassan@mans.edu.eg

QINGKAI KONG

Department of Mathematics

Northern IllinoIs University

DeKalB, IL 60115, USA

E-mail address: kong@math.niu.edu 